\title{
The Low-Background HPGE $\Gamma$-Spectrometer OBELIX for the Investigation of the Double Beta Decay to Excited States
}

\author{
V.B. Brudanin ${ }^{1}$, V.G. Egorov ${ }^{1}$, R. Hodák ${ }^{2}$, A.A. Klimenko ${ }^{1}$, P. Loaiza ${ }^{3}$, \\ F. Mamedov ${ }^{2}$, F. Piquemal ${ }^{3}$, E. Rukhadze ${ }^{2}$, N.I. Rukhadze ${ }^{1}$, I. Stekl ${ }^{2}$, \\ Yu.A. Shitov ${ }^{1}$, G. Warot ${ }^{3}$, E.A. Yakushev and M. Zampaolo ${ }^{3}$ \\ '(Joint Institute for Nuclear Research, Russia) \\ ${ }_{2}^{2}$ (Institute of Experimental and Applied Physics, Czech Technical University in Prague, Czech Republic) \\ ${ }^{3}$ (Laboratoire Souterrain de Modane, France)
}

\begin{abstract}
A new ultra-low-background spectrometer based on a HPGe detector with a sensitive volume of 600 $\mathrm{cm}^{3}$ was developed to investigate rare nuclear processes, such as resonant neutrino-less double electron capture $(0 v E C / E C)$ and double beta decay processes $\left(2 \vee 2 \beta, 2 v \beta^{+} E C, 2 v E C / E C\right)$ to the excited states of daughter nuclei. The spectrometer was installed at the Modane underground laboratory (LSM, France, $4800 \mathrm{~m}$ w.e.). Sensitivity of the spectrometer and its background were tested. A new method for the efficiency calibration in measurements of low-active samples was developed. A spectrometer was used for the measurements of low active materials and samples. Results obtained in $395 \mathrm{~h}$ investigation of resonant OvEC/EC decay of ${ }^{106} \mathrm{Cd}$ to the $2718 \mathrm{keV}$ and $2741 \mathrm{keV}$ excited states of ${ }^{106} \mathrm{Pd}$ with $\sim 23.2 \mathrm{~g}$ of enriched ${ }^{106} \mathrm{Cd}$, $\beta^{+} E C, E C / E C$ decays of ${ }^{58} \mathrm{Ni}$ in measurements of $\sim 21.7 \mathrm{~kg}$ sample of natural nickel and $2 \mathrm{~V} 2 \beta$ decay of ${ }^{100} \mathrm{Mo}$ sample with the mass of $2588 \mathrm{~g}$ to the $0^{+}, 1130 \mathrm{keV}$ and $2^{+}, 539.5 \mathrm{keV}$ excited states of ${ }^{100} \mathrm{Ru}$ are presented.
\end{abstract}

Keywords: double beta decay, gamma-ray spectrometry, HPGE detector.

\section{Introduction}

Investigation of the neutrino-less double beta decay $\left(\beta^{-} \beta^{-}, \beta^{+} \beta^{+}, \beta^{+} E C, E C / E C\right)$ is of great importance in particle and nuclear physics as it is a powerful tool for studying the properties of neutrino and weak interactions. The study of neutrino-less double beta $(0 v \beta \beta)$ decay may clarify nature of the neutrino (Majorana or Dirac), absolute neutrino mass scale, the neutrino mass hierarchy (normal, inverted, or quasi degenerate) and $\mathrm{CP}$ violation in the lepton sector. Interest in studying the double-beta decay to excited states of daughter nuclei has grown recently, due to the search for the resonant neutrino-less double electron capture within some nuclei [1] and the investigation of two-neutrino double beta decay to excited states of daughter nuclei. Such processes are accompanied by emission of $\gamma$-quanta in the de-excitation of excited states. These $\gamma$-quanta may be detected by low background HPGe spectrometers with high efficiency and good energy resolution. The main candidates in studying of double beta decay processes using low background HPGe spectrometers are resonant neutrinoless double electron capture $(0 \mathrm{vEC} / E C)$ in ${ }^{106} \mathrm{Cd} \rightarrow{ }^{106} \mathrm{Pd}$ decay $(K L, 2741 \mathrm{keV} ; K K, 2718 \mathrm{keV} ; K L, 2737 \mathrm{keV}$; etc.) [2], two neutrino double beta $\left(2 \mathrm{~V} 2 \beta^{-}\right)$decay ${ }^{100} \mathrm{Mo} \rightarrow{ }^{100} \mathrm{Ru}$ to excited states $\left(0^{+}{ }_{1}, 1130 \mathrm{keV}[3]\right.$ and $2^{+}, 540$ $\mathrm{keV}), 2 \mathrm{~V} \beta^{+} \mathrm{EC}$ and $2 \mathrm{VEC} / \mathrm{EC}$ decays of ${ }^{58} \mathrm{Ni} \rightarrow{ }^{58} \mathrm{Fe}\left(2^{+}, 811 \mathrm{keV}\right)$.

HPGe spectrometers used for such measurements must be produced from materials with extremely low radioactive contamination and have high sensitive volume. In this work, we present the new ultra-lowbackground HPGe spectrometer OBELIX located at the Modane underground laboratory and used for the investigation of rare nuclear processes. To obtain the detector efficiency for such measurements, the original method of using special low-active samples with known mass and activity was developed. Capabilities of the spectrometer and the method of detector efficiency calibration are shown for the investigations of double beta decays of ${ }^{100} \mathrm{Mo},{ }^{106} \mathrm{Cd}$ and ${ }^{58} \mathrm{Ni}$.

\section{Design of The Spectrometer}

The low background HPGe detector, OBELIX, is produced by the company Canberra, and is based on P-type crystal with the sensitive volume of $600 \mathrm{~cm}^{3}$. The mass of the detector is approximately $3 \mathrm{~kg}$ and the detector relative efficiency is $160 \%$. The crystal was mounted in ultra-low background U-type cryostat with a cooled FET (Field Effect Transistor). The energy resolution (FWHM) of the detector is $\sim 1.2 \mathrm{keV}$ at $122 \mathrm{keV}$ $\left({ }^{57} \mathrm{Co}\right)$ and $\sim 2 \mathrm{keV}$ at $1332 \mathrm{keV}\left({ }^{60} \mathrm{Co}\right)$. The measured value of Peak to Compton ratio is up to 113 . To achieve the maximum efficiency for registration of the external radiation, the germanium crystal is placed at a minimum distance $(4 \mathrm{~mm})$ from the entrance window of the end cap of the aluminum cryostat, and the thickness of the entrance window and walls of the end cap is less than $1.6 \mathrm{~mm}$. To minimize the detector background, the design of the internal part of the Canberra cryostat for such detectors was modified in our common activity with 
Canberra (France). All constructive details of the cryostat were produced from materials with ultra-low radioactive contaminations. All materials used in the inner part of the cryostat were selected on the base of results obtained in measurements performed with low-background spectrometers of Modane underground laboratory (LSM, France, $4800 \mathrm{~m}$ w.e.).

The HPGe crystal is mounted in a holder made of Al-Si (4\%) alloy specially selected for its high radiopurity. This special low $\mathrm{Z}$ alloy has been chosen since it is almost free of cosmogenic activity, and has only $0.38_{-0.14}^{+0.19} \mathrm{mBq} / \mathrm{kg}$ of ${ }^{26} \mathrm{Al}$. However, the main drawback is the thorium contamination at the level of 1.4 $\mathrm{mBq} / \mathrm{kg}$. All other parts inside the holder are made of plastic or lead-free brass. In particular, all screws are homemade from lead-free brass. A measurement of lead-free brass showed the activity of ${ }^{210} \mathrm{~Pb}<100 \mathrm{mBq} / \mathrm{kg}$ (see Table 1). The first stage of preamplifier with FET is placed below the holder and gives one of the main contributions to the internal background of the detector. To decrease radioactive background from FET we added a $10 \mathrm{~mm}$ thick roman lead (activity of $<60 \mathrm{mBq} / \mathrm{kg}$ [4]) cylinder at the bottom of the holder to shield the crystal against the radioactivity of the FET. The end cap of the cryostat is made of the same Al-Si alloy as the crystal holder. It is sealed to the bottom part by an O-ring, made from viton (which, even containing high radioactivity levels, gives minor contributions since it is placed far from the crystal). Table 1 gives the radioactive contaminations of some materials used in the cryostat construction, measured by gamma spectrometry. All measurements were performed at LSM, except the aluminum alloy measured at LNGS with the GeMPI detector [5].

Table 1. Radioactive contaminations of materials used in the cryostat construction.

\begin{tabular}{|l|c|c|c|c|c|}
\hline Material & \multicolumn{7}{|c|}{ Radioactivity of materials $[\mathrm{mBq} / \mathrm{kg}]$} \\
\hline Al- Si alloy & Ra-226 & Ra-228 & Th-228 & K-40 & Others \\
\hline Lead-free brass & $0.27 \pm 0.19$ & $<0.11$ & $1.4 \pm 0.2$ & $1.1_{-0.1}^{+0.2}$ & $\mathrm{Al}-26: 0.38_{-0.14}^{+0.19}$ \\
\hline FET support* & $4.9 \pm 1.3$ & $<5$ & $<3$ & $<40$ & $\mathrm{~Pb}-210:<100$ \\
\hline O-ring & $1.6 \pm 0.2$ & $2.9 \pm 0.6$ & $<0.5$ & $<5$ & $\mathrm{Bi}-207: 1.7 \pm 0.7$ \\
\hline Roman lead & $910 \pm 80$ & $320 \pm 70$ & $350 \pm 30$ & $1360 \pm 400$ & \\
\hline
\end{tabular}

*The radioactivity levels in the FET support are given in $\mathrm{mBq} / \mathrm{unit}$.

The detector part of the cryostat is encircled by the passive shielding of several layers of roman lead $(\mathrm{PbI})$ with a total thickness of $\sim 12 \mathrm{~cm}$ (activity of $<60 \mathrm{mBq} / \mathrm{kg}$ ) and low active (PbII) lead (activity of $\sim 5-20$ $\mathrm{Bq} / \mathrm{kg}$ [4]) with a total thickness of $\sim 20 \mathrm{~cm}$ and placed inside a tightly closed stainless steel cover (see Figure 1). To measure big samples (up to 10 l) in Marinelli beakers and bobbins, two internal layers of roman lead with a thickness of $\sim 7 \mathrm{~cm}$ can be removed. To decrease the concentration of ${ }^{222} \mathrm{Rn}$ gas near the detector the passive shielding of the spectrometer is flushed with radon-depleted air (concentration of ${ }^{222} \mathrm{Rn}$ is $\sim 15 \mathrm{mBq} / \mathrm{m}^{3}$ ) from a radon trapping facility installed in LSM. The electronic part of the spectrometer is based on NIM electronic modules produced by Canberra. A 3106D High Voltage module of Canberra supplies a voltage $+6000 \mathrm{~V}$ to the detector. Signals from the preamplifier PSC761 coupled to the detector part are passing through the Spectroscopy Amplifier 2022 and then digitizing by 16384 channels ADC Multiport II. The software used for data taking and analysis of spectra is Genie 2000 version 3.2.1. The spectrometer is installed at the Modane underground laboratory (LSM, France, $4800 \mathrm{~m}$ w.e.), which provides the suppression of a muon flux in $2 \times 10^{6}$ times and fast neutrons in $\sim 10^{3}$ times.

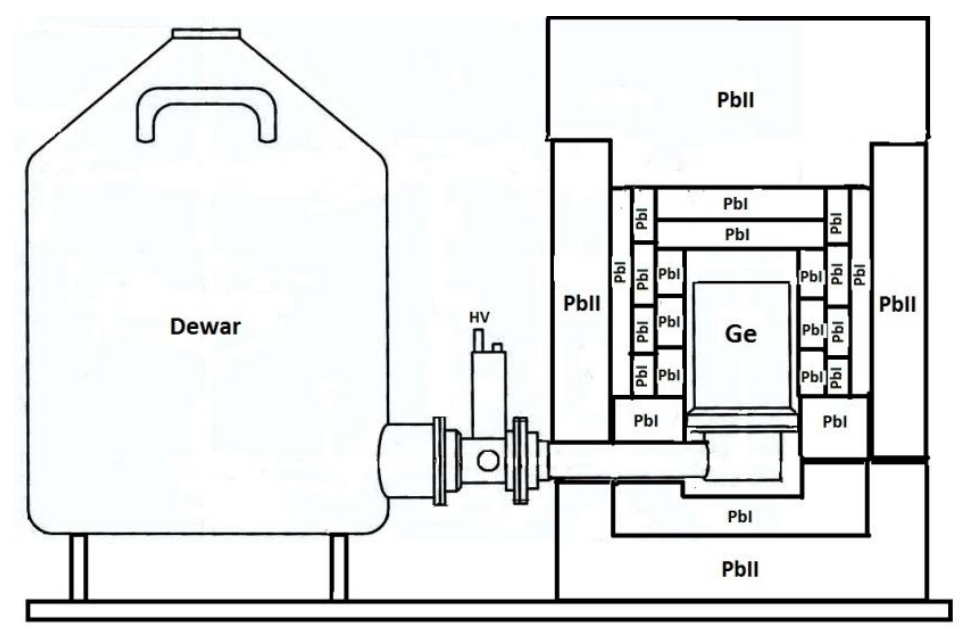

Fig.1. HPGe detector in a passive shielding. 


\subsection{Geometries of measurements}

\section{Low Background Measurements}

The low-background measurements are based on the comparison of the measurements of samples and the spectrometer background in a given geometry. The possible measurement configurations are the following (see Figure 2): i) completely assembled passive shielding for measurements of samples in small plastic boxes, placed on the end cap of the cryostat. The volume available for samples in this geometry is $\varnothing 120 \mathrm{~mm}$ and a height of $50 \mathrm{~mm}$; ii) the shielding with removed first layer of the internal part of the passive shielding around the detector with a thickness of $36 \mathrm{~mm}$, and two layers of shielding under the detector with a total thickness of $64 \mathrm{~mm}$. This geometry is used for measurements of samples in home made 1.7 L Marinelli bobbins and small $(2.2 \mathrm{~L})$ Marinelli beakers, produced from delrin; iii) the shielding with removed first layer and part of the second layer (without last lead ring) of the internal part of the passive shielding. The geometry is used for measurements of samples in big industrial Marinelly beakers (4L, model 448G-E, produced from polypropylene) [6]; iv) completely removed two internal layers of the internal part of the passive shielding. The geometry is used for measurements of specially produced bulk samples (for example, sample of natural nickel). The usual duration of long-term measurement is about one month. Background measurements are usually followed by measurements of the corresponding samples.

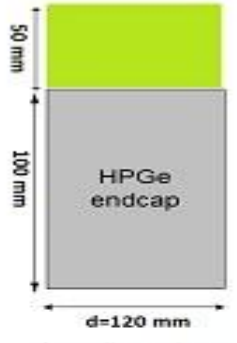

a) Configuration !

$0-255 \mathrm{~mm}$

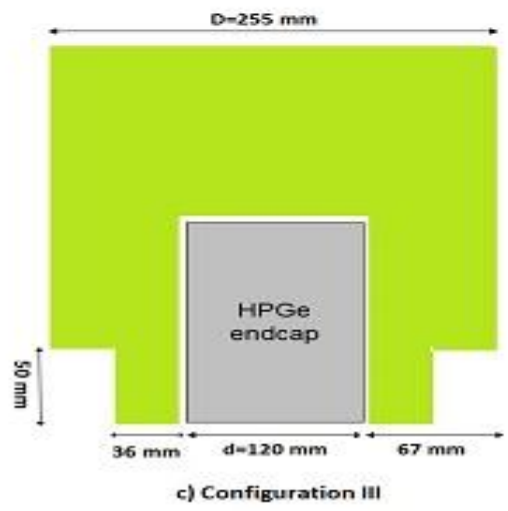

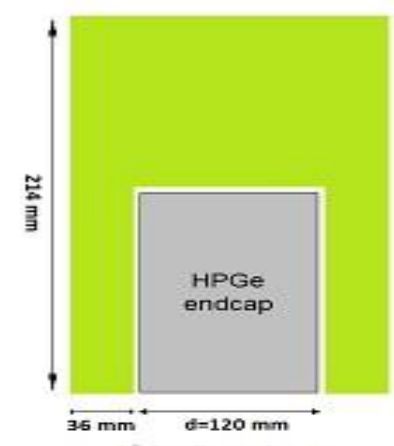

b) Configuration "I

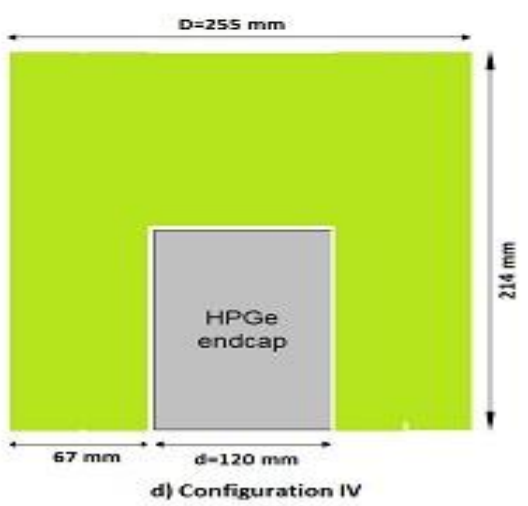

Fig.2. Possible geometries of measurements.

\subsection{Background of the OBELIX detector}

The detector was produced recently and its background consists of not only natural activity of ${ }^{40} \mathrm{~K}$, and isotopes of $\mathrm{U}$ and $\mathrm{Th}$ radioactive series, but also of some short living cosmogenic isotopes, such as ${ }^{57} \mathrm{Co}$ $\left(\mathrm{T}_{1 / 2}=271.79 \mathrm{~d}\right),{ }^{54} \mathrm{Mn}\left(\mathrm{T}_{1 / 2}=312.3 \mathrm{~d}\right),{ }^{65} \mathrm{Zn}\left(\mathrm{T}_{1 / 2}=244.26 \mathrm{~d}\right)$ produced in a germanium crystal during its location on the Earth's surface. Starting the detector was installed in LSM underground laboratory (November 2010), the level of the above mentioned isotopes permanently decreases and in few years the traces of these isotopes will not be observed in the background spectra (see Table.2). The integral count rate in the region of 40-3000 keV obtained from the measurement of the detector background in September 2011 (Figure 3, down spectrum) was 173 counts/kg·d and in September 2013 (Figure 3, upper spectrum) was 73 counts $/ \mathrm{kg} \cdot \mathrm{d}$. The radioactive background is the object of our permanent control to avoid any contaminations of the detector and the passive shielding. 

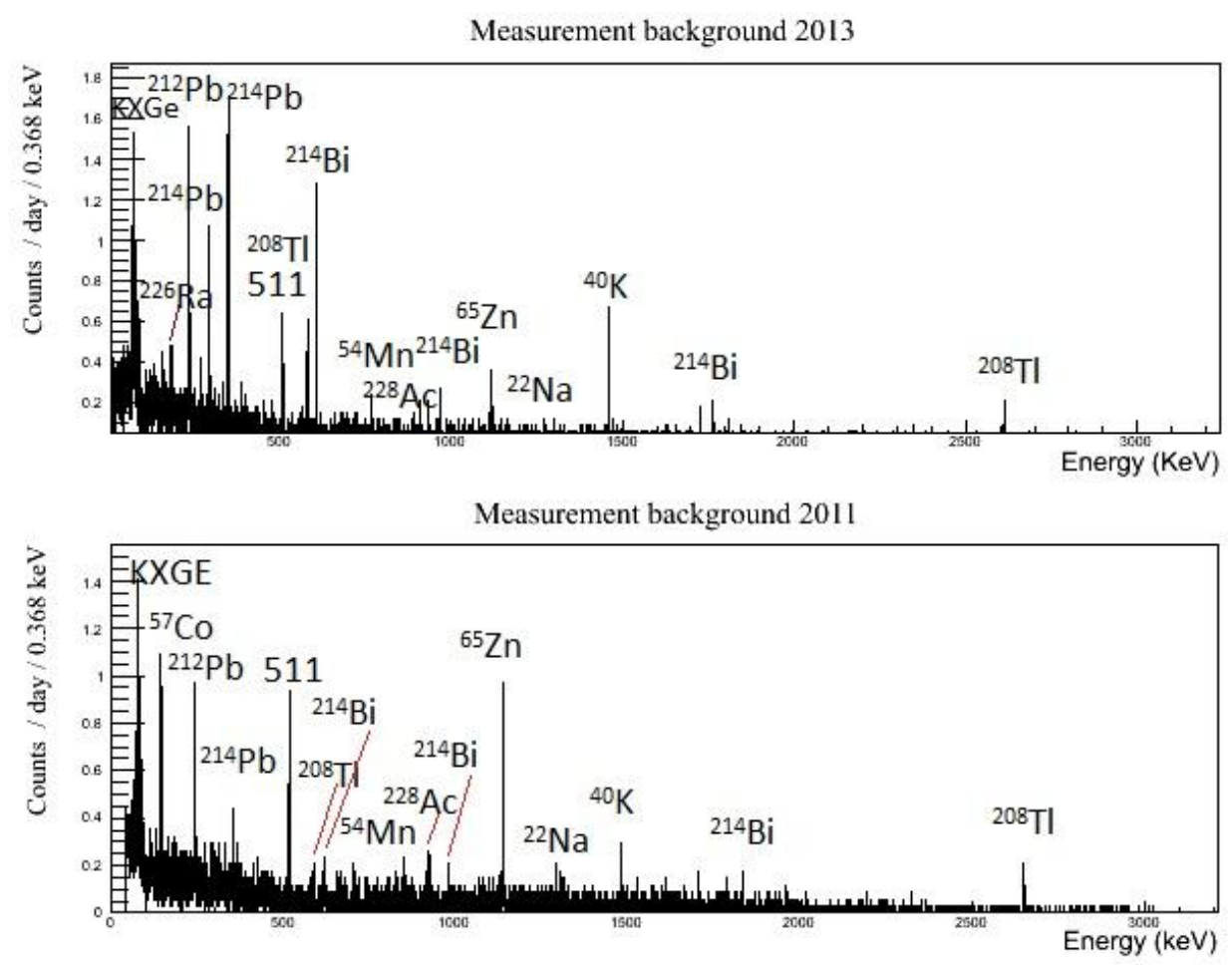

Fig.3. Background spectra of HPGe detector measured in September 2011 (top) and in September 2013 (bottom).

Table 2: Background count rates of natural activity of the OBELIX HPGe detector.

\begin{tabular}{|c|c|c|}
\hline $\mathrm{E}[\mathrm{keV}]$ & Nuclide & Counts/kg day \\
\hline 238 & ${ }^{212} \mathrm{~Pb}$ (Th chain) & $0.60 \pm 0.25$ \\
\hline 295 & ${ }^{214} \mathrm{~Pb}$ (U chain) & $0.24 \pm 0.05$ \\
\hline 352 & ${ }^{214} \mathrm{~Pb}$ (U chain) & $0.20 \pm 0.09$ \\
\hline 583 & ${ }^{208} \mathrm{Tl}$ (Th chain) & $0.18 \pm 0.07$ \\
\hline 609 & ${ }^{214} \mathrm{~B}$ (U chain)i & $0.21 \pm 0.06$ \\
\hline 911 & ${ }^{228} \mathrm{Ac}$ (Th chain) & $0.25 \pm 0.05$ \\
\hline 969 & ${ }^{228} \mathrm{Ac}$ (Th chain) & $0.20 \pm 0.04$ \\
\hline 1460 & ${ }^{40} \mathrm{~K}$ & $0.34 \pm 0.05$ \\
\hline 2615 & ${ }^{208} \mathrm{Tl}($ Th chain) & $0.17 \pm 0.06$ \\
\hline
\end{tabular}

\subsection{Efficiency of measurements}

Energy calibration of the spectrometer is performed by using pointed radioactive sources ${ }^{152} \mathrm{Eu}$ and ${ }^{133} \mathrm{Ba}$. To obtain the detector efficiency, except already named pointed sources, the specially prepared lowactive samples with known mass and activity were used. The samples were produced from the powder of $\mathrm{La}_{2} \mathrm{O}_{3}$, and from the mixture of some powdery filler and the powder of $\mathrm{La}_{2} \mathrm{O}_{3}$. The natural $\mathrm{La}$ in this powder consists of $0.0888 \pm 0.0007 \%$ of ${ }^{138} \mathrm{La}$ with a half-life of $\mathrm{T}_{1 / 2}=(1.02 \pm 0.01) \times 10^{11}$ years [7], which is characterized by the emission of $\mathrm{Y}$-rays with energies of $788.7 \mathrm{keV}$ and $1435.8 \mathrm{keV}$. The $\mathrm{La}_{2} \mathrm{O}_{3}$ powder was chosen for producing calibration samples due to its low specific and volume activity. This allows to produce low active calibration samples (tens of $\mathrm{Bq}$ ) in Marinelli beakers while similar standard samples have the activity of $5 \mathrm{kBq}$ and higher. This leads to the increasing errors during calibration, especially for detectors with high efficiency. Several measurements were performed with samples prepared on the base of $\mathrm{La}_{2} \mathrm{O}_{3}$ powder, with activities in the range of $\sim 7$ to $\sim 300 \mathrm{~Bq}$ to approach as much as possible the geometries of measurements of investigated lowbackground samples. In some of them, $\mathrm{La}_{2} \mathrm{O}_{3}$ powder was measured in small plastic boxes, in others $\mathrm{La}_{2} \mathrm{O}_{3}$ powder was mixed with some other non-radioactive powders, such as flour, and measured in Marinelli beakers and bobbins. The homogeneity of mixtures of powders was determined by a monochrome color of final mixtures (mixed powders had different colors), and was tested by measurements of specific activities of small amounts of the mixture (in plastic boxes), placed on the top of the end cap of the detector. Based on the results obtained in measurements of $\mathrm{La}_{2} \mathrm{O}_{3}$ and with standard pointed sources of ${ }^{152} \mathrm{Eu}$ and ${ }^{133} \mathrm{Ba}$, efficiency curves for the measurements of double beta emitters in several "standard" geometries were obtained (see Figure 4). These results are in a good agreement with Monte Carlo (MC) simulations. For example, to obtain the efficiency for the real measurement of metallic foil of enriched ${ }^{100} \mathrm{Mo}$, which was placed around end cap in the Marinelli

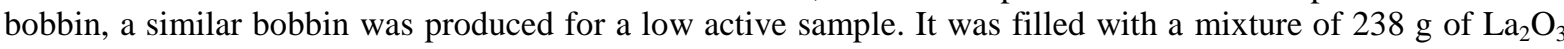


powder and $954 \mathrm{~g}$ of flour and measured in the same geometry as enriched ${ }^{100}$ Mo during $1800 \mathrm{~s}$. Given the mass of $\mathrm{La}_{2} \mathrm{O}_{3}$ it was easy to calculate the activity of the sample (activity $168 \pm 2 \mathrm{~Bq}$ ), and the yield of gamma quanta of corresponding energies to determine the spectrometer efficiency. The accuracy of results based on this method should be better than $10 \%$. The efficiency curve (Exp.) based on measurements of a low active sample (black points) was used to test the accuracy of MC simulations performed on the base of GEANT 4 and GEANT 3. Figure 5 shows the experimentally measured efficiency curve in comparison with several Monte Carlo simulations. The results of such experimental calibration are useful to determine the accuracy of MC simulation in low-background measurements.

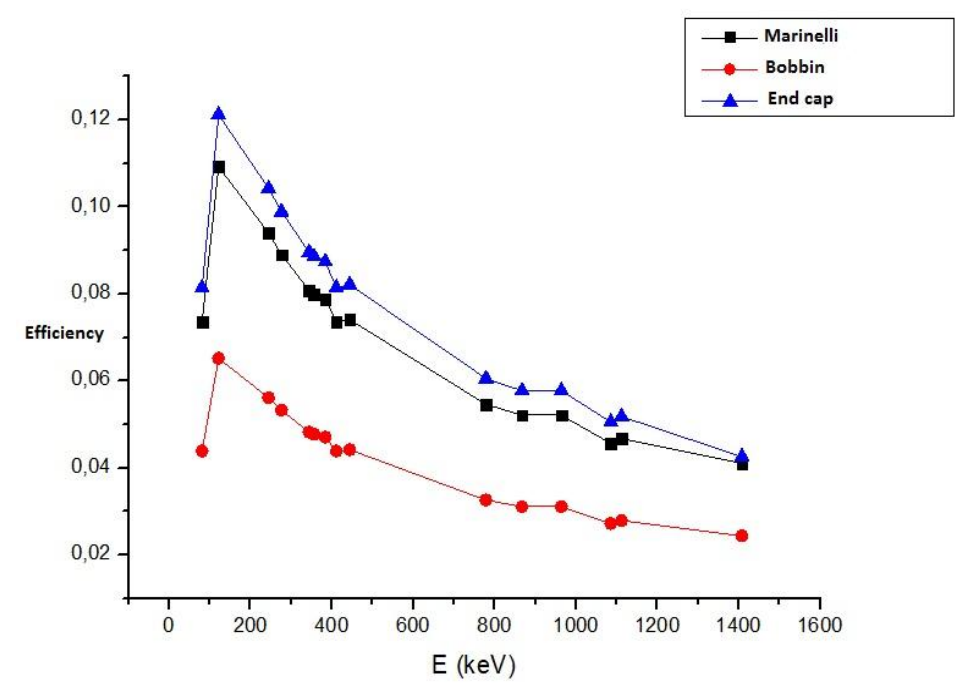

Fig.4. Efficiency curves for some "standard" geometries.

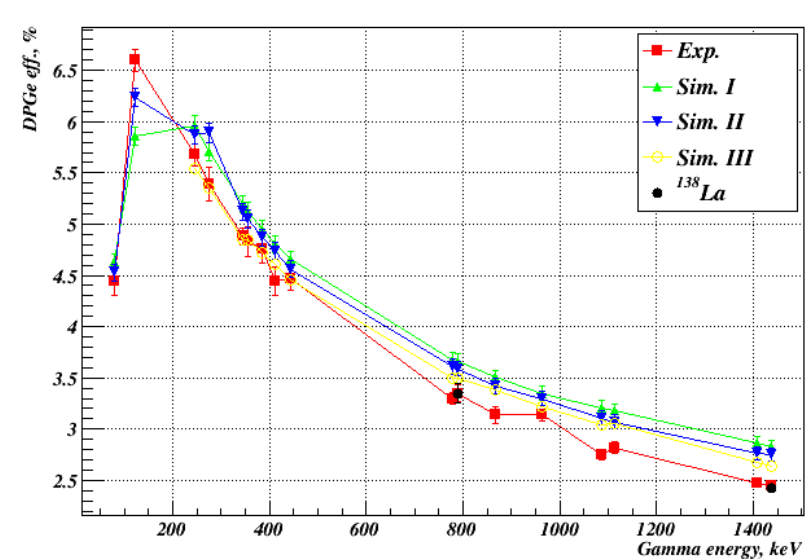

Fig.5. Experimental (Exp) and calculated (Sim) efficiency curves of the HPGe spectrometer for measurement of Marinelli bobbin.

\subsection{Measurements of low-active samples}

Several long-term measurements were performed to obtain activities of materials used in constructions of low-background detectors, such as SuperNemo Demonstrator, TGV-2 and Edelweiss. The results obtained for selected samples are presented in a Table.3. Upper limits in the table are given at 95.45\% CL, according to [8].

Table.3. Results of measurements of materials, used in low-background spectrometers.

\begin{tabular}{|l|l|l|l|l|l|l|l|}
\hline Material & \multirow{2}{*}{$\begin{array}{l}\text { Mass } \\
\end{array}$} & \multirow{2}{*}{$\begin{array}{l}\text { Time } \\
{[\text { Days }]}\end{array}$} & \multicolumn{5}{|c|}{ Activity $[\mathrm{mBq} / \mathrm{kg}]$} \\
\cline { 4 - 8 } & & Ra-226 & Th-228 & K-40 & Co-60 & Others \\
\hline Polyethylene & 3.9 & 28 & $0.65 \pm 0.08$ & $0.30 \pm 0.07$ & $<1$ & $<0.06$ & U235: $<0.13$ \\
\hline Pure Iron & 0.6 & 20 & $0.5 \pm 0.3$ & $<0.9$ & $<5$ & $0.34 \pm 0.12$ & Mn54: $1.3 \pm 0.2$ \\
\hline Delrin & 0.9 & 8 & $2.5 \pm 0.5$ & $2.8 \pm 0.8$ & $5.6 \pm 1.5$ & & \\
\hline
\end{tabular}




\section{Investigation of Double Beta Decay To Excited States}

The sensitivity of the spectrometer OBELIX allows us to measure rare nuclear processes, such as resonant neutrino-less double electron capture $(0 \mathrm{vEC} / \mathrm{EC})$ of ${ }^{106} \mathrm{Cd}$ and $2 \mathrm{~V} 2 \beta^{-}$decay of ${ }^{100} \mathrm{Mo}$ to excited states of the daughter nuclei. The sample of ${ }^{100}$ Mo used in the NEMO-3 experiment [9], [10] was measured with the OBELIX spectrometer to test its radiopurity after the end of the experiment. The sample was metallic foil of ${ }^{100} \mathrm{Mo}$ with the mass of $2588 \mathrm{~g}$ and enrichment of $99.8 \%$. It was placed in a Marinelli bobbin around the end cap of the detector and measured during $2288 \mathrm{~h}$. Taking into account the high sensitivity of the spectrometer OBELIX we were able to investigate simultaneously the $2 \mathrm{~V} 2 \beta^{-}$decay of ${ }^{100} \mathrm{Mo}$ to the $0^{+}, 1130 \mathrm{keV}$ and $2^{+}, 539.5$ $\mathrm{keV}$ excited states of ${ }^{100} \mathrm{Ru}$. Two peaks with energies $539.5 \mathrm{KeV}$ and $590.8 \mathrm{keV}$ were clearly visible in the spectrum. Calculation of these peaks allowed us to obtain the most precise value of the half-life of the investigated process $-\left(2 \mathrm{v} \beta \beta\right.$ decay of ${ }^{100} \mathrm{Mo}$ to $0_{1}^{+}$excited state of ${ }^{100} \mathrm{Ru}-T_{1 / 2}=[7.5 \pm 0.6$ (stat. $) \pm$ 0.6 (sys.) $\times 1020 y(90 \% \mathrm{CL})$. The obtained results were the subject of another paper [11], which were published in the frame of NEMO-3 collaboration.

Investigation of the $0 \mathrm{vEC} / \mathrm{EC}$ resonant capture of ${ }^{106} \mathrm{Cd}$ was performed also with the detector OBELIX and metallic ${ }^{106} \mathrm{Cd}$ with a mass of $23.166 \mathrm{~g}$ and enrichment of $99.57 \%$. The measured sample consisted of 16 circle foils of enriched ${ }^{106} \mathrm{Cd}$, each with a diameter of $52 \mathrm{~mm}$ and a thickness of $70 \pm 10 \mathrm{mg} / \mathrm{cm}^{2}$. They were stacked in a Teflon box and placed on the top of the detector end cap (upper efficiency curve of Figure 4). The test measurement with ${ }^{106} \mathrm{Cd}$ (see Figure 6) lasted only $395 \mathrm{~h}(\sim 16.5 \mathrm{~d}$ ) giving us almost the same limit on the resonant $0 \mathrm{v} E C / E C$ decay of ${ }^{106} \mathrm{Cd}$, as it was obtained in a $12900 \mathrm{~h}$ measurement with TGV-2 spectrometer [12]$\mathrm{T}_{1 / 2}(\mathrm{KK}, 2718 \mathrm{keV})>1.4 \times 10^{20} \mathrm{y}, \mathrm{T}_{1 / 2}(\mathrm{KL}, 2741 \mathrm{keV})>0.9 \times 10^{20} \mathrm{y}$. The sensitivity of the OBELIX spectrometer allows us to reach the level of $\mathrm{T}_{1 / 2}>10^{21} \mathrm{y}$ in 4 months of such a measurement for KK capture to $2718 \mathrm{keV}$ excited state of ${ }^{106} \mathrm{Pd}$ and in 6 months for KL capture to $2741 \mathrm{keV}$ excited state of ${ }^{106} \mathrm{Pd}$. Such longterm investigation will be performed in the near future.

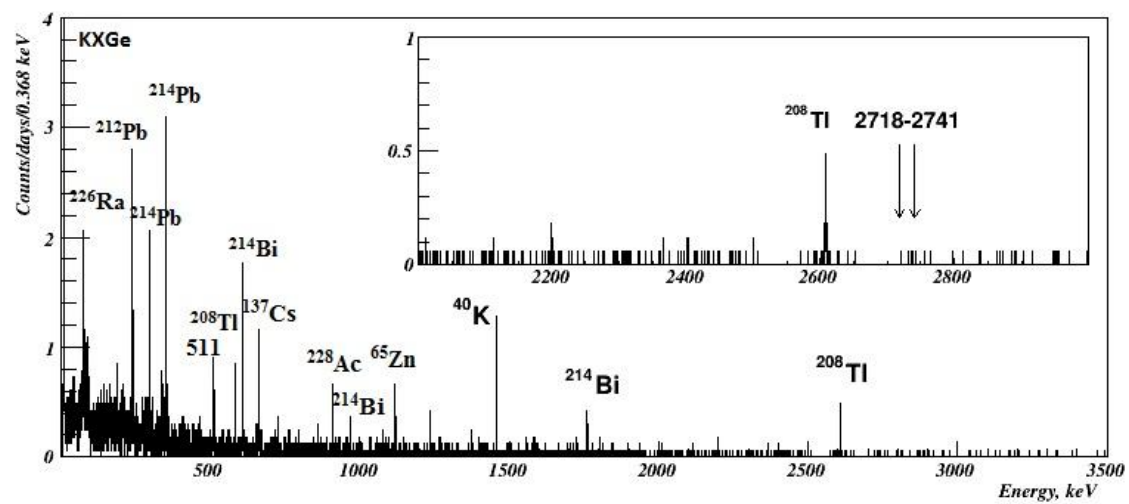

Fig.6. Spectrum of ${ }^{106} \mathrm{Cd}$ and ROI of the spectrum measured with the OBELIX detector.

The investigations of double beta decay $\left(\beta^{+} \mathrm{EC}, \mathrm{EC} / \mathrm{EC}\right)$ of ${ }^{58} \mathrm{Ni}$ with the OBELIX detector were performed at LSM (Modane). The sample of natural Ni, containing $\sim 68 \%$ of ${ }^{58} \mathrm{Ni}$ with a total mass of $\sim 21.7 \mathrm{~kg}$ was prepared in a shape of a Marinelli beaker and was placed on the OBELIX detector. Two experimental runs were performed with the investigation samples. The first run was started at the middle of October 2014 and lasted 47.5 days. The main goal of this measurement was to obtain radioactive contaminations of the investigated sample. The main contaminations in measured sample were ${ }^{57} \mathrm{Co}$ and ${ }^{58} \mathrm{Co}$ (see Table 4). These activities were produced in the sample during its location on the Earth's surface. Their activities were found to be high for a long-term measurement of ${ }^{58} \mathrm{Ni}$.

After one year, during which the sample was placed in LSM, another experimental run was performed. This test run was started in September 2015 and lasted only 19 days. The results obtained during this measurement showed the decreasing of the activity of ${ }^{58} \mathrm{Co}$ in the region of interest by about one order of magnitude. The region of interest (ROI) of the energy spectrum obtained in these measurements for the investigation of $0 \mathrm{vEC} / \mathrm{EC}$ decay of ${ }^{58} \mathrm{Ni}$ with radiative decay of $1918.3 \mathrm{keV}$ [13] is shown on Figure 7. Based on the data accumulated in these two test measurements, the preliminary experimental limits (all at 90\% CL) on $\beta^{+} \mathrm{EC}$ and EC/EC decay of ${ }^{58} \mathrm{Ni}$ were obtained $-T_{1 / 2}\left(2 v \beta^{+} E C, 0^{+} \rightarrow 0^{+}\right)>1.2 \times 10^{22} y, T_{1 / 2}\left(2 v \beta^{+} E C, 0^{+} \rightarrow\right.$ $2+, 811 \mathrm{keV}>4.0 \times 1021$ y, , T122vECEC, $0+\rightarrow 2+, 811 \mathrm{keV}>4.6 \times 1021 \mathrm{y},$, T120vEC/EC, $0+\rightarrow 0+>2.2$ $\times 10^{22} y$, improving previous experimental limits [14] and [13].

The long-term investigation of $\mathrm{Ni}$ sample is planned to be performed in 2017, when the background from ${ }^{58} \mathrm{Co}$ and ${ }^{57} \mathrm{Co}$ isotopes will be negligible. 
Table 4. Radioactive contaminations in the sample of natural nickel.

\begin{tabular}{|c|c|c|}
\hline Isotope & Activity $[\mathrm{mBq} / \mathrm{kg}]$ & Half-life $\left[\mathrm{T}_{1 / 2}\right]$ \\
\hline${ }^{54} \mathrm{Mn}$ & 0.73 & $312.3 \mathrm{~d}$ \\
\hline${ }^{56} \mathrm{Co}$ & 2.3 & $77.3 \mathrm{~d}$ \\
\hline${ }^{57} \mathrm{Co}$ & 5.0 & $271.8 \mathrm{~d}$ \\
\hline${ }^{58} \mathrm{Co}$ & 3.83 & $70.9 \mathrm{~d}$ \\
\hline${ }^{60} \mathrm{Co}$ & 0.22 & $5.272 \mathrm{y}$ \\
\hline
\end{tabular}

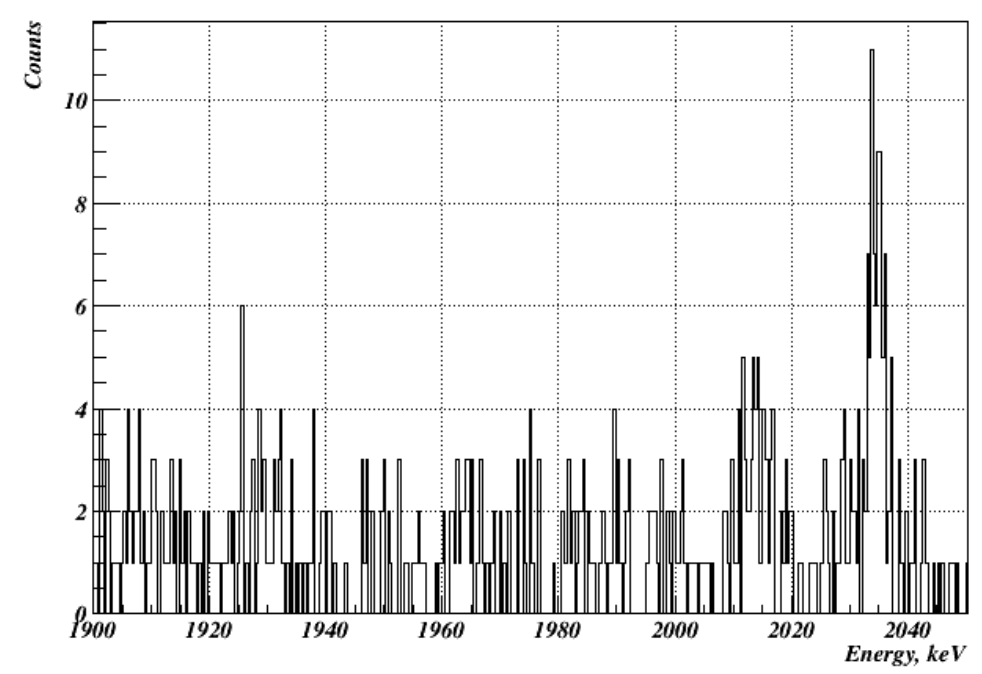

Fig.7. The ROI of the energy spectrum of ${ }^{58} \mathrm{Ni}$ for the investigation of $0 \mathrm{vEC} / \mathrm{EC}$ decay of ${ }^{58} \mathrm{Ni}$ with the radiative decay of $1918.3 \mathrm{keV}$.

\section{Conclusion}

An ultra-low background HPGe spectrometer, OBELIX, was developed and installed at the Laboratoire Souterrain de Modane. Due to careful selection of all of the spectrometer components and using the shielding from low active materials, low intrinsic background of the spectrometer was achieved. A new original method for the determination of efficiency for low-background measurements, based on using, in addition with the "standard" radioactive sources, specially produced low-active samples with known mass and activity, was developed and successfully used in the investigations with the OBELIX detector. Investigation of rare nuclear processes, such as $0 \mathrm{vEC} / \mathrm{EC}$ resonant capture of ${ }^{106} \mathrm{Cd}$ and $\beta^{+} \mathrm{EC}$, EC/EC decay of ${ }^{58} \mathrm{Ni}$ was performed with the OBELIX spectrometer, resulting in new experimental limits on these modes. The measurement with ${ }^{100} \mathrm{Mo}$ sample was performed with highest sensitivity, energy resolution and good S/B ratio, leading to the most precise value in determination of the half-life of this transition. The investigations of $\beta \beta$ decay with the OBELIX spectrometer will be continued with ${ }^{58} \mathrm{Ni},{ }^{82} \mathrm{Se},{ }^{124} \mathrm{Sn},{ }^{96} \mathrm{Zn},{ }^{106} \mathrm{Cd}$ and ${ }^{150} \mathrm{Nd}$.

\section{Acknowledgements}

This work was performed under LEA-JOULE collaboration agreement and IN2P3-JINR collaboration agreements No.15-93 and partly supported by grants MSM6840770029, RI LSM/ULISSE, LG11030 and by Russian Foundation for Basic Research under grant No.14-02-00568. We would like to thank the LSM staff for their technical assistance.

\section{References}

[1]. W. C. Haxton and G. S. Stephenson, "Jr. Double beta decay," Prog.Part.Nucl.Phys, vol. 12, pp. 409 -479, 1984.

[2]. V. I. Tretyak and Y. G. Zdesenko, "At. Data and Nucl. Data Tables," vol. 80, p. 83, 2002.

[3]. A. S. Barabash and al., "Two neutrino double-beta decay of 100Mo to the first excited 0+ state in 100Ru," Phys. Lett., B, vol. 345, pp. 408-413, 1995.

[4]. P. Loaiza and al., "Low background germanium planar detector for gamma-ray spectrometry," NIM A, vol. 634, p. 64, 2011.

[5]. G. Heusser, M. Laubenstein and H. Neder, "Radioactivity in the environment, International Conf. on Isotopes in Environmental Studies, Aquatic forum 2004, 25.-29. october 2004," in Radionuclides in the Environment, Amstedram, Elsevier, 2006 , p. 495.

[6]. "http://www.marinellibecher.com/en/products/products_marinellibeaker.html," [Online].

[7]. A. A. Sonzogni, Nuclear Data Sheets for A = 138, vol. 98, p. 515, 2003.

[8]. C. Hurtgen, S. Jerome and M. Woods, "Revisiting Currie - how low can you go?," Appl. Radiation and Isotopes, vol. 53, p. 45, 2000 .

[9]. $\quad$ R. Arnold and al., "First Results of the Search for Neutrinoless Double-Beta Decay with the NEMO 3 Detector," Phys. Rev. Lett., vol. 95, p. 182302, 2005. 
[10]. R. Arnold and al., "Chemical purification of molybdenum samples for the NEMO 3 experiment," Nucl. Phys. A, vol. 781, p. 209, 2007.

[11]. R. Arnold and al., "Investigation of double beta decay of 100Mo to excited states of 100Ru," Nucl. Phys. A, vol. 925, p. $25,2014$.

[12]. N. I. Rukhadze and al., "Experiment TGV-2. Search for double beta decay of 106Cd," Jour. of Phys.: Conf. Ser., vol. 375, p. 042020, 2012.

[13]. B. Lehnert and al., " A search for the radiative neutrinoless double electron capture of 58Ni," no. arXiv:1605.03287v1 [nucl-ex], 2015.

[14]. S. I. Vasilev and al., JETP Lett., vol. 57, p. 631, 1993. 